\title{
Discovering the Early Earth from the Oldest Rock Records
}

\section{VICKIE C. BENNETT}

The Australian National University

Presenting Author: vickie.bennett@anu.edu.au

This year marks 50 years since the publication of the landmark paper of Black et al. (1971, EPSL) which provided the first direct radiometric evidence for an ancient ( $\geq 3.6$ billion years old) rock record and dispelling prevailing notions of the time that the young Earth was far too dynamic for rocks from its first billion years to survive. After the initial discovery it rapidly emerged how 'normal' this early Earth appeared to be, with a retained hydrosphere (oceans) by $3.8 \mathrm{Ga}$ and continental crust dominated by rather ordinary granitic rocks. We now recognize rocks of this age from nine areas of varying extent distributed worldwide and although they comprise only one millionth of Earth's surface, Eoarchean to Hadean rocks and minerals are direct samples of early Earth chemistry and conditions; combined with increasingly sophisticated analytical approaches they provide the ground-truth for models of early terrestrial planet formation, environments and evolution.

In the early 2000's a significant milestone in investigations of earliest Earth was the ability to measure subtle (typically less than $15 \mathrm{ppm}$ ) isotopic differences produced by chemical processes occurring during the lifetime of now extinct parent nuclides, in particular ${ }^{146} \mathrm{Sm}$ (half-life $=103 \mathrm{myr}$ ) and ${ }^{182} \mathrm{Hf}$ (halflife $=8.9$ myr). The resultant small isotopic anomalies in ${ }^{142} \mathrm{Nd}$ and ${ }^{182} \mathrm{~W}$, as compared with signatures in modern rocks, are now observed throughout early Archean terranes, recording the timing of differentiation events in the first $<400$ million years of Earth history, enabling direct comparisons with lunar and meteorite events, and serving as powerful tracers of mantle dynamics.

Despite increasingly detailed investigations the style of tectonic processes operative on the early Earth continues to be vigorously debated. Emerging multiple lines of observations, however, argue that convergent tectonic processes were producing new continental crust by at least $3.8 \mathrm{Ga}$. The existence of early "plate tectonics" takes on added significance in considering the possible necessity of efficient chemical recycling between the surface and the deep Earth for creating habitable conditions.

In this talk I will present some past highlights, current controversies and emerging work in the rapidly evolving field of early Earth investigations. 